\title{
ESTABLISHING AND MAINTAINING SWARDS ON AIRSTRIPS ON FARMS IN THE AUCKLAND PROVINCE
}

By E. H. ARNOLD, Assistant Fields Superintendent, Department of Agriculture, Auckland

In 1955-56, 303 aircraft made 1,183,794 flights from over 8000 airstrips to drop 404,933 tons of fertiliser, 4401 tons of fencing materials and rabbit poison, as well as 374,811 gallons of spray. In the early days of aerial farming light aircraft were successfully flown from paddocks or airstrips where little grading had been done or not much-attention given-to provide-a hardwearing sward. More recently with the use of newer types of aircraft lifting heavier loads, permanent airstrips have been constructed, some at considerable expense and in many cases to serve a number of neighbouring farmers. Up to 1000 tons of fertiliser may be flown off an airstrip in a year.

The progress of aerial farming has been both rapid and spectacular. Increased quantities of fertiliser are being dropped on sheep and cattle farms on hill country and on fat lamb farms on rolling country. Even dairy farmers on undulating country are turning to aerial topdressing. Spring topdressing, the dropping of rabbit poison, fencing material, and other farm supplies, and aerial spraying all result in working from an airstrip under less favourable conditions than when topdressing is done in the autumn from a dry and load-bearing surface. Airstrips must be efficient under a wide range of conditions. They are efficient only when, if flying conditions are favourable, the maximbm flying hours are put in and the maximum tonnage is flown off. A poor airstrip means lighter loads per take-off and fewer take-offs per hour. This makes aerial farming more costly. Further, and of vital importance, the strip must be safe for pilots to use.

To determine the present condition of farm airstrips in the Auckland Province I conducted a survey of 132 strips. These were selected at random from Maungaturoto to the King Country and Bay of Plenty. The survey has been confined to surfaces and swards and did not include siting, freedom from obstructions, and other factors influencing flying operations. Defects in surface and sward are closely connected. Many are increasing the costs of 
aerial work and creating hazards for pilots. Any surface or sward defect narrows down the effective width of the strip. A loaded 'plane once it begins its run to take off cannot change direction while still in contact with the ground.

Poor and inadequate construction causes many defects.

Table 1-Surface Defects (Mainly of Construction) on 132 Airstrips.

$\begin{array}{llr}\text { Graded unevenly } & & 69 \\ \text { Hills and hollows } & & 66 \\ \text { Corrugations } & & 41 \\ \text { Side slope } & \ldots . . & 10 \\ \text { Hollow centre } & \ldots .- & 6 \\ \text { Not consolidated } & \ldots . . & 24 \\ \text { Wet places } & & 44 \\ \text { Soft places } & \ldots . . . & 47\end{array}$

Sixty-nine strips were graded unevenly. This may be due to the use of narrow working implements. A wide "leveller" such as is used for grading irrigation lands or one made from a couple of railway rails would eliminate much of this, particularly those longitudinal marks left to start water down the strip and cause erosion. Cultivation marks and implement wheel marks can turn a 'plane sideways with fatal results.

Humps and hollows found on 66 strips vary in magnitude. The humps dry out, lose the cover of turf, and produce dust conditions. The hollows can collect water, creating soft patches which must be avoided by aircraft when taking off. Hills and hollows can turn into waves or corrugations.

Corrugations occuring on the 41 strips may have been left when construction is skimped. On others they have developed rapidly from unevenly graded surfaces with hills and hollows. The effect of a corrugated road on a car is well known; the effect of a corrugated airstrip on aircraft can be imagined.

Side slope present on 10 strips usually results where a strip is cut along the side of a hill, leaving half the formation on the solid and half on a filling which settles sideways excessively. On some strips excavation is not complete enough to produce a level cross surface. Side slope is very dangerous to aircraft, causing them to run off sideways.

A hollow centre as noted on six strips results from either inadequate levelling or through aircraft taking off down the same track on an unconsolidated strip. Water collects and, running down, causes erosion of the surface.

Twenty-four strips were not consolidated. This lack of consolidation causes many other defects. A delay of 12 months before sowing the seed increases consolidation and allows defects to be observed and remedied. 
Wet and soft places on 44 and 47 strips respectively result from localised uneven filling, grading, and consolidation, as well as from poorly drained areas within the strip.

Most strips examined in the survey required no attention to draining. Out of the 49 requiring drainage, 15 need major work, 12 some work, and 22 had already been effectively drained. No matter what the longitudinal slope is, the surface of the strip should be almost flat or level across, or with a slight crown of not more than 6in. on a strip 2 chains wide. This crown does not affect the take-off of aircraft, yet drains off surface water.

Many surface defects occur after construction.

$\begin{array}{ccc}\text { Table 2-Surface Defects (Post Construction) } & \text { on } 132 \text { Airstrips. } \\ & & \text { Strips } \\ \text { Gullying and erosion-transverse } & 27 \\ & \text {-longitudinal } & 53 \\ \text { Tractor, truck, and implement damage } & 37 \\ \text { Stock tracks } & & 45 \\ \text { Po a ching and pugging } & \text {... } & 40 \\ \text { Rabbit-hotes }\end{array}$

Any uncontrolled movement of surface water causes damage to the surface, to fillings, and to cuttings. Transverse and longitudinal gullying of the surface is often rapid and severe. On 86 strips with fillings, on only 54 had the sides been successfully grassed. Attempts to grass the sides on another 13 had failed, resulting in varying stages of erosion. No attempts had been made to grass the sides of fillings on 16 strips. These were severely eroded. On three, fillings were held by planting pampas grass or trees and shrubs kept cut low.

Table 3-Condition of Cuttings on 55 Strips.

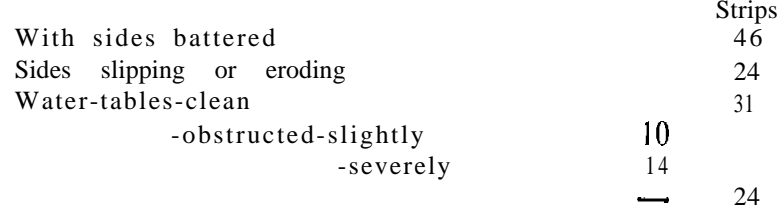

Of the 55 strips with cuttings, only 46 had the sides properly battered. On 24 the sides were eroding and slipping.

Of the water-tables, 31 were clean, IO were slightly obstructed, and 14 severely blocked. Debris accumulating in the water-tables forces surface water to spread over the strip with all the accompanying damage. Some farmers themselves are not exempt from damaging airstrips. They use them as farm roadways. In dry weather little harm is done, but during wet conditions serious 


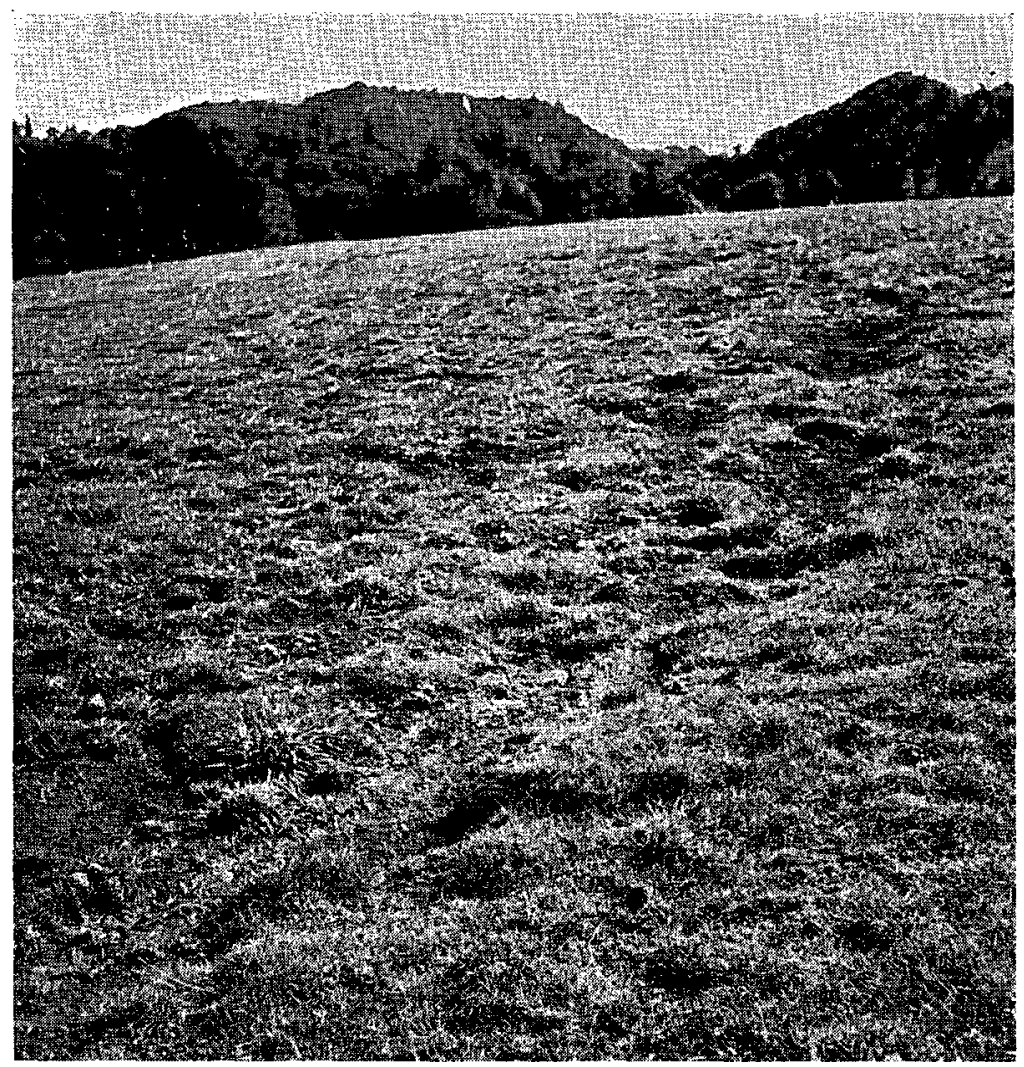

Some erosion damage on the sides of an airstrip.

damage results. Trucks, tractors, and stock moving from field to field are best kept off the strip.

Next to erosion, most trouble is caused by stock. With sheep, grazing tracks are formed where gateways and water troughs arc badly placed. Despite the fact that 91 airstrips were grazed by cattle, such a practice is most undesirable. Forty strips had seriously pugged or poached surfaces which, when drying out, produce irregularities most damaging to the structure of aircraft. Twenty-three strips were cut for hay. This practice, an undesirable one, opens up the sward and changes its composition. Hay was fed out on 43 strips and this is associated with cattle grazing, including pugging under wet conditions. Uneaten hay and cattle droppings result in uneven fertility and uneven swards. Sometimes seedy hay is fed out to get seed in to bare patches and thin, open swards. 
The seed is mostly of undesirable species. It is better practice to prepare the surface and resow poor areas.

Grazing by sheep alone is most efficient. A large mob needs to be put on, the growth eaten down rapidly, the sheep removed, and the area spelled. In other words use the flock as a lawnmower. Present grazing management of airstrips is almost universally inefficient. Of the 132 strips surveyed, only 19 were fenced as units so that grazing could be controlled. The area necessary ranges from 5 to 12 acres, depending on where the fences need to be erected to protect cuttings and fillings. Thirtyseven strips had been constructed across a number of fields and removable fences are placed across the strips when they are not in use. Dissimilar grazing of each field creates sward and surface differences, while ridges and tracks are formed by stock alongside the fence line.

Damage can occur to surfaces and swards during flying operations. On 77 strips aircraft were loaded on the runway or take-off areas with damage of varying magnitude. Spilt fertiliser caused damage, some quite severe, on 52 strips. No details were recorded of damage by loaders and this was considerable on some strips. Loading areas off the side or turning circles at the end obviate such trouble.

Table 4-Dominant Sward Constituents.

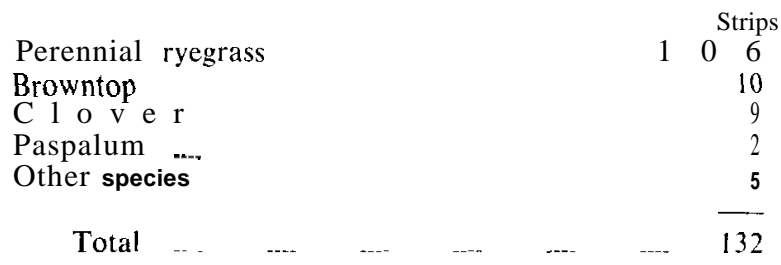

Little consideration has been given to the seed mixtures sown. In most cases a general-purpose pasture mixture for grazing animals has been used. On a few strips nature was left to sow the seed to cover the surface. While browntop is dominant on 10 strips only and present in varying amounts on 41 other strips, its use has not been exploited as it should be. On 40 strips large amounts of Poa annua were present. Thus it is not surprising that 50 per cent. of the strips would be dusty or muddy durin' flying operations.

Table S-The Major Sward Faults Found on 132 Airstrips.

Can be classed as-

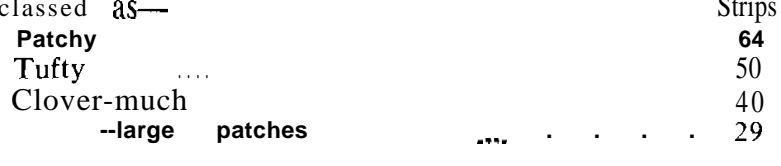

G.8 
Ryegrass, cocksfoot, and Yorkshire fog can become patchy and tufty, and with wind and rain causing soil erosion between the tufts, a very bumpy surface results. This is dangerous to aircraft taking off, damaging to aircraft structure, and hazardous to pilots.

Clover is not required in airstrip swards. It should not be encouraged or even sown. Much clover, even if evenly distributed, reduces the braking capacity of the surface, particularly when the turf is moist or wet. Large patches, greasy and slippery when moist, have been known to cause skids, and it is on record as causing the wreck of a 'plane.

A suitable seed mixture for most areas in the Auckland Province is:

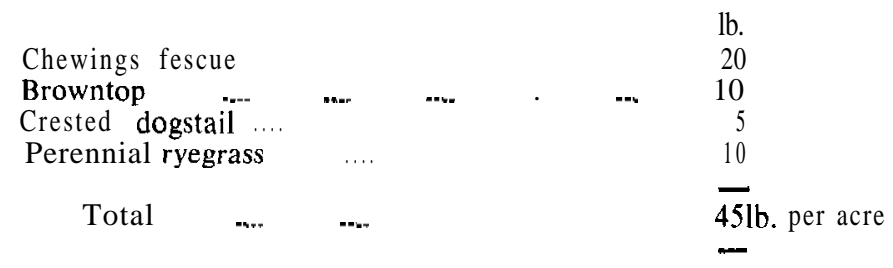

This can be increased to 60lb. per acre round and within a chain or two of the loading area and on those poor soil areas on the very steep portions of some strips where extra fertiliser is also necessary to obtain a good sward. With dressings of superphosphate and sulphate of ammonia, this mixture will form a hard-wearing sward with a good build-up of turf mat. Such a turf does best on a somewhat acid soil, one with a $\mathrm{pH}$ of about 5.0. Dressings of lime and basic slag will encourage clovers. The former will increase the $\mathrm{pH}$ to a figure more favourable to luscious, non-wearing species. Work at Palmerston North has shown that a soil with a low $\mathrm{pH}$ has more stability than one with a high $\mathrm{pH}$.

$\mathrm{pH}$ tests on 120 airstrips gave the following results:

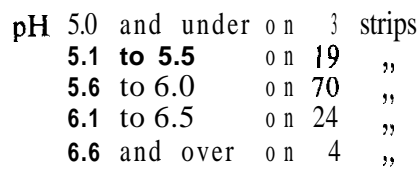

These figures are more indicative of grazing pastures rather than airstrip swards. Much improvement is possible if attention is given to eliminating clovers and making conditions suitable for hard-wearing, browntop-dominant swards.

An airstrip should provide an even, well-graded surface some 
400 yards long and 2 chains wide, whatever its slope is along its length. On poorly draining soil a slight crown is advisable, but otherwise the surface across should be level. A desirable cover of turf forming browntop and chewings fescue without clover can be maintained with use of superphosphate and sulphate of ammonia. Time is required to build up a turf, especially on the steeper places generally of hard subsoil. On these places immediate attention is necessary to repair defects during establishment. After sowing it will often be advantageous to delay using a strip until a turf is built up all over. Immediately seasonal operations are complete, the strip should be dressed with soil to fill hollows, wheel and skid marks, and worn places. These should be rolled down firmly, resown, topdressed, and not stocked until after the grass strikes. The strip should not be used as a farm road.

Of the 132 airstrips surveyed, on 68 it would not have been possible to drive a medium or heavy car safely at 40 or more miles per hour. On 56 per cent. of the strips-major-repairs, even to reconstruction, resurfacing, or regrassing, are necessary. On another 30 per cent. some improvements are required, leaving only 14 per cent. in reasonable order.

Good, efficient airstrips enable large tonnages to be flown off each season, keep down repairs to aircraft, save pilots' lives, and reduce the cost of aerial farming to landowners. An example of this is quoted:

Before a strip was properly laid down and treated it cost $£ 318 \mathrm{~s}$. 6d. per ton to fly fertiliser off. Two years later, after it had been reconstructed properly, it cost $£ 25 \mathrm{~s}$, a ton. Thus the farmer saved $£ 1 \mathrm{I} 3 \mathrm{~s}$. 6d. for every ton flown off. A group of farmers flying off 1000 tons would save well over $£ 1500$.

\section{DISCUSSION}

Q. Is there any inspection required by the Civil Aviation Department of farm airstrips?

A. No. This is left to air operators and farmers to decide whether the strip is workable.

The matter was further amplified by $\mathrm{Mr} \mathrm{R}$. Scott who intimated that there was at present no control by the Civil Aviation Department of farm airstrips.

Q. Has $\mathrm{Mr}$ Arnold any figures relating to the use of heavier aircraft on farm airstrips and any damage they are doing?

A. I have no data with me, but the opcrators have figures of the weight loading for various types of aircraft.

$\mathrm{Mr}$ E. A. Madden: There are now some 4000 airstrips in New Zealand and some of these are sub-standard. None is under the control of the Civil Aviation Authority. It is left to the pilots to take care and precautions and determine whether they are prepared to fly off the strip. This does not apply to heavy aircraft which must use licensed 
aerodromes. I think that 40 miles per hour is perhaps too high a standard to set for farm airstrips and that 30 miles per hour only is necessary. If a strip is wavy and corrugated the plane will have to travel further before it can take off. Whereas on a good surface only $100 y d s$. may be required, where the surface is poorer $140 y d s$. will probably be required. A small amount of side slip does not matter. I $\frac{1}{2} \mathrm{ft}$ in $100 \mathrm{ft}$. is considered not objectionable. In my opinion clover was more dangerous previously than it is at present, because today the tread pattern of aircraft tyres can partly overcome the previous disadvantages of any clover being present. 1 think that $11 \mathrm{~b}$. of white clover in the mixture could be very desirable from the point of pasture thrift. Also browntop should be used in the mixture. In normal topdressing practice in farm airstrips, a good deal of fertiliser is spilt along the strip. Therefore I consider a dressing of sulphate of ammonia would be all that is required. Accordingly $\mathrm{I}$ am asking $\mathrm{Mr}$ Arnold two questions:

Q.1. Why does he recommend the use of phosphate, and

Q.2. Will he please discuss what he thinks of the inclusion of paspalum in the airstrip mixture in warm regions.

A. Firstly, I am convinced that on the results of the survey the standard of 40 miles per hour for a medium heavy car to be driven over the airstrip is not too high. While the pilot has to decide whether he will use the strip, there is no doubt that better strips can save lives and reduce the costs of putting manure on the farm. Good strips reduce cost of repairs to aircraft. This will mean ultimately that with good strips aerial operators can reduce the costs of applying the fertiliser. The experience of aerial topdressing firms and pilots is that clover is not required. The necessary nitrogen can be applied to the sward by sulphate of ammonia. As regards my recommendation to topdress with super the whole of the airstrip, the evidence showed that superphosphate dropped from planes is too heavy in some places and others get nothing. This results in a very uneven sward. Therefore I consider it necessary to use a dressing of superphosphate over the whole strip. If clover becomes a problem, methods such as $\mathrm{Mr}$ Matthews has outlined (hormone treatment) may be required. On the point of using paspalum in the mixture, if it is well distributed in the sward, it is quite all right, otherwise it may become very tufted and pilots don't like this

Q. Would $\mathrm{Mr}$ Arnold please comment on the ability of larger planes to fly off strips where a 30 miles per hour standard has been fixed'?

A. The aircraft operators say 40 miles per hour is a reasonable standard and none of them wants less.

$\mathrm{Mr}$ Robertson (A commercial aircraft operator): I think $\mathrm{Mr}$ Arnold is to be commended for the data he has collected. I would support him in everything he has said. The 40 miles per hour standard is little enough particularly for the lighter constructed aircraft. I would like to see it raised to 50 miles per hour. 